\title{
Phase transitions of octamethylcyclotetrasiloxane confined inside aluminosilicate and silicate nanoporous matrices
}

\author{
Angelina Sterczyńska $\cdot$ Anna Deryło-Marczewska • \\ Małgorzata Śliwińska-Bartkowiak · Julia Z. Piotrowska • \\ Marcin Jarek • Kamila Domin
}

Received: 3 April 2014/ Accepted: 30 June 2014/Published online: 1 August 2014

(C) The Author(s) 2014. This article is published with open access at Springerlink.com

\begin{abstract}
We report the melting behaviour of a dipolar cyclic siloxane liquid: octamethylcyclotetrasiloxane (OMCTS) confined in three mesoporous silica matrices: AlSBA-15, SBA-15 and CPG glasses, using differential scanning calorimetry and dielectric spectroscopy. We investigate the influence of acid sites on the adsorptive properties of mesoporous silica materials, which were synthesized by applying Pluronic-type polymers as porecreating agents. Aluminosilicate matrices have been synthesized by direct synthesis procedure using aluminium chloride. These materials characterized by $\mathrm{N}_{2}$ sorption measurements, and the small-angle X-ray scattering data exhibit the same hexagonal P6 $\mathrm{mm}$ structure with a mean mesopores size of $4.6 \mathrm{~nm}$ (Al-SBA-15) and $4.9 \mathrm{~nm}$ (SBA-15). The controlled pore glasses used in this experiment have pores of mean diameter of $7.5 \mathrm{~nm}$. For all systems studied, the OMCTS melting point in pores has been found to decrease with decreasing pore diameter. This
\end{abstract}

A. Sterczyńska · M. Jarek · K. Domin

The NanoBioMedical Centre, Umultowska 85, 61-614 Poznan, Poland

A. Sterczyńska · M. Śliwińska-Bartkowiak (凹) ·

J. Z. Piotrowska $\cdot$ K. Domin

Faculty of Physics, Adam Mickiewicz University,

Umultowska 85, 61-614 Poznan, Poland

e-mail: msb@amu.edu.pl

A. Deryło-Marczewska

Faculty of Chemistry, Maria Curie Sklodowska University, 20-031 Lublin, Poland

J. Z. Piotrowska

Institute of Physics, Poznan University of Technology,

Nieszawska 13A, 60-965 Poznan, Poland result is in qualitative agreement with that obtained in molecular simulation where the adsorbate-wall interactions are weak compared to the adsorbate-adsorbate interactions.

Keywords Confinement effect .

Octamethylcyclotetrasiloxane (OMCTS) · Plastic crystal · Maxwell-Wagner relaxation - Wettability parameter $\alpha_{w}$

\section{Introduction}

Phases confined in nanopores often exhibit a physical and chemical behaviour that is significantly different from that of the bulk phase. These effects arise from reduced dimensionality and from the strong interaction of the adsorbate molecules with the walls of the porous materials [1-5]. Examples of such effects are shifts in phase boundaries, the presence of new surface-driven phases, heats of adsorption, enhanced pressures, selective adsorption in the case of mixtures and shifts in chemical equilibrium for reactions. These effects are relevant to applications such as the purification of water and air streams, heterogeneous catalysis, drug delivery, sensors, energy storage, the fabrication of nanomaterials such as nanowires and electrodes for fuel cells. An examination of the contributions of the adsorbate-adsorbate and adsorbatewall interactions to the description of the melting behaviour of confined systems, the wetting parameter $\alpha_{\mathrm{w}}$ defined as the ratio of the effective average solid-fluid to fluidfluid interaction strength, was introduced [6]. The large value of $\alpha_{\mathrm{w}}$ corresponds to the well-wetting systems, when $\alpha_{\mathrm{w}}$ tends to 0 - the systems are practically non-wetting. Walls with $\alpha_{w}$ values much greater than one can be regarded as "strongly attractive" and an elevation of melting temperature of confined system relatively to the 
bulk is expected, while those with $\alpha_{\mathrm{w}}$ much less than one can be considered "weakly attractive" and the depression of the melting point of such systems relatively to the bulk is observed. The case of well wetting system, characterized by small contact angle $\left(\theta=18^{\circ}\right)$, is the octamethylcyclotetrasiloxane $(\mathrm{SiO})_{4}\left(\mathrm{CH}_{3}\right)_{8}$, OMCTS, placed on silica surface.

So far, OMCTS was investigated from the point of view of surface force apparatus (SFA) experiments, where molecularly thin films of this liquid were placed between mica surfaces; the results have been discussed in terms of first-order transitions to solid-like or glasslike behaviour in these films [7-9]. During shear of mica surfaces separated by films of OMCTS one to three molecular layers thick, the confinement-induced transitions between liquid-like and solid-like response were found [10].

The octamethylcyclotetrasiloxane $(\mathrm{SiO})_{4}\left(\mathrm{CH}_{3}\right)_{8}$ is a cyclic molecule where four silicon and four oxygen atoms form an inner ring. To every $\mathrm{Si}$ atom, two methyl groups are bonded. These eight $\mathrm{CH}_{3}$ groups build the outer 'shell' of the molecule whose dipole moment equals to $1.09 \mathrm{D}$. The overall shape of an OMCTS molecule is described as almost spherical, but somewhat compressed in one dimension with a $0.9 \mathrm{~nm}$ of diameter. Due to its relatively large size and near-spherical shape, OMCTS is considered as a good large-scale model of a simple Lennard-Jones liquid. In this paper, the melting behaviour of OMCTS placed on silica glasses: SBA-15 of mesopores size of $4.9 \mathrm{~nm}$ and Al-SBA-15, where Al is incorporated into the lattice of SBA-15 with the pore size of $4.6 \mathrm{~nm}$, is discussed. For investigation of influence of the matrix structure and kind of pore wall on the fluid-wall interactions, the melting behaviour of OMCTS placed in CPG of pore size of $7.5 \mathrm{~nm}$ is also presented.

Al-SBA-15 silica glasses were synthesized using modified method described by Bhange et al. [11], and SBA-15 was synthesized by modified procedure described by Joo et al. [12]. The porosity characterization was performed by nitrogen adsorption/desorption isotherms at 77 K using ASAP 2020 analyzer (Micromeritics Corp.). The structure determination of the synthesized materials was obtained using small-angle X-ray scattering (SAXS), EDS, TEM and SEM methods.

\section{Experimental}

Synthesis and characterization of Al-SBA-15 and SBA-15

The formation of $\mathrm{Si}-\mathrm{O}-\mathrm{Al}$ linkages leads to isomorphous substitution of $\mathrm{Si}^{+4}$ by some $\mathrm{Al}^{+3}$ ions. By adjusting the $\mathrm{H}_{2} \mathrm{O} / \mathrm{HCl}$ molar ratio, $\mathrm{Al}$ gets incorporated into the lattice of SBA-15, which is evidenced by SAXS, EDS, $\mathrm{N}_{2}$ adsorption and pore size distribution, TEM and SEM data.

Purely siliceous molecular sieve materials have an electrically neutral framework and consequently no acid sites because they have only silanol groups on their surface, which are of low acid strength and catalytically non-active. Incorporation of heteroatoms, such as Al into microporous zeolites, will introduce a charge imbalance in the framework which is balanced by protons, and generating bridging hydroxyl groups (SiOHAl, Broensted acid sites). This substitution can modify the composition of the inorganic walls. Here, aluminium is incorporated into the framework of a silicate material by direct synthesis procedure.

\section{Materials}

Pluronics [PE 6400- $\left.(\mathrm{EO})_{13}(\mathrm{PO})_{70}(\mathrm{EO})_{13}\right]$ was obtained from BASF (Poland). Tetraethyl orthosilicate (TEOS $98 \%$ ) was purchased from ALDRICH. Hydrochloric acid (35-38 \%, analytical grade) and aluminium chloride $\mathrm{AlCl}_{3}$ were purchased from PCR (Polish Chemical Reagents, Poland).

\section{Preparation of SBA-15}

A sample of polymer-templated 2D hexagonally ordered silica was synthesized by modified procedure described by Joo et al. [12]. We used non-ionic triblock copolymer Pluronic PE 6400 instead of classical surfactant Pluronic P123 and moreover, we have modified the ageing conditions. The silica sample was obtained using TEOS as a silica source under acidic conditions. A typical synthesis procedure was as follows: $4.0 \mathrm{~g}$ of PE 6400 was dissolved in $150 \mathrm{~g}$ of $1.6 \mathrm{M} \mathrm{HCl}$ solution. To this solution, $8.50 \mathrm{~g}$ of TEOS was added, and the resulting mixture was stirred at $313 \mathrm{~K}$ for $24 \mathrm{~h}$. The ageing process was conducted in autoclave under static condition at elevated temperature, at $393 \mathrm{~K}$ for $24 \mathrm{~h}$. The crystallized product was filtered off, washed with bidistilled water, dried and calcined in air at $873 \mathrm{~K}$ for $6 \mathrm{~h}$.

Preparation of Al-SBA-15 by direct synthesis procedure

The synthesis followed a procedure being a modification of the method described by Bhange et al. [11]. We used nonionic triblock copolymer Pluronic PE 6400 instead of classical surfactant Pluronic P123 as a structure-directing agent. Aluminium was incorporated into the framework of a silicate material through direct synthesis in which an aluminium precursor is added into gel prior to hydrothermal process. In a typical procedure, $4 \mathrm{~g}$ of copolymer was dissolved in $30 \mathrm{~mL}$ of water. After stirring for $2 \mathrm{~h}$, a clear 
(a)

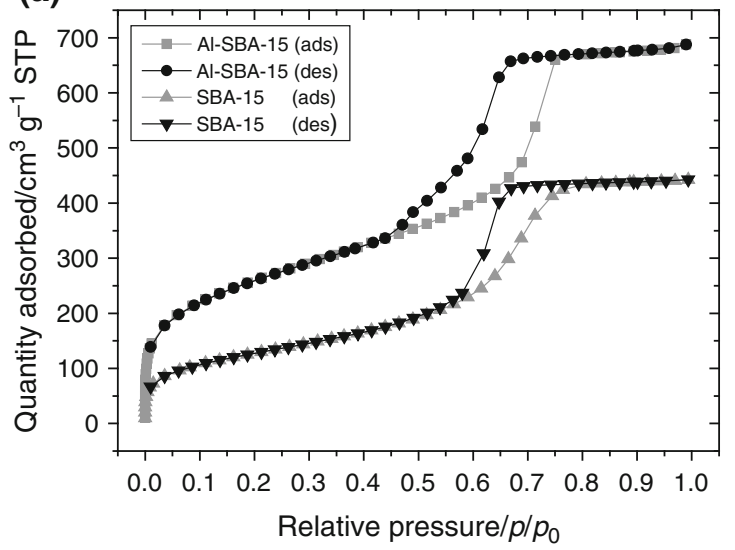

(b)

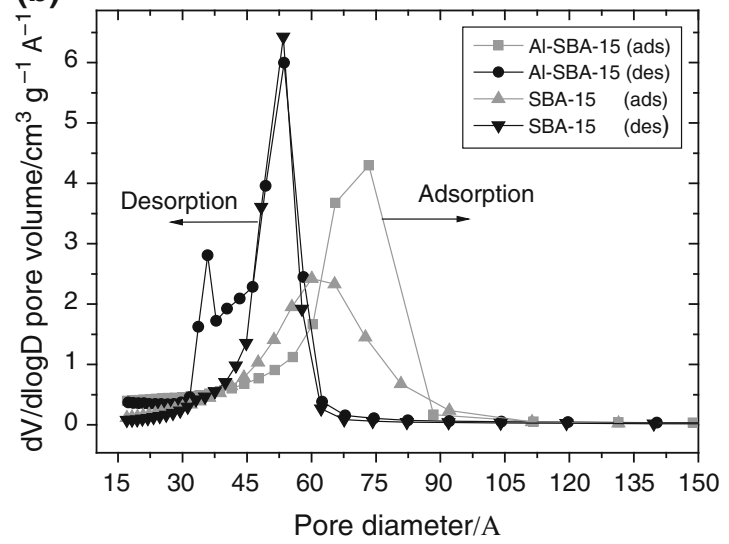

Fig. 1 a Nitrogen adsorption/desorption isotherms and b pore size distributions for the mesoporous Al-SBA-15 and SBA-15 silica

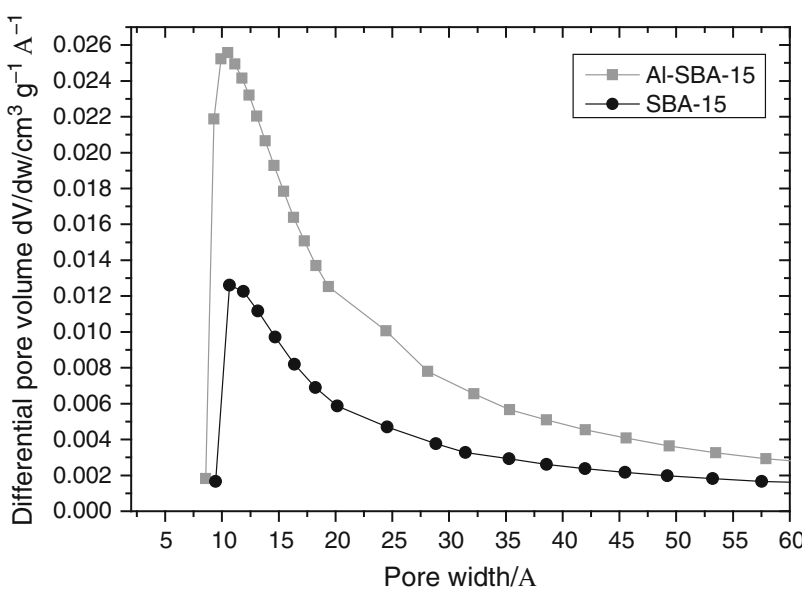

Fig. 2 Horvath-Kawazoe distribution of micropores volume for the Al-SBA-15 and SBA-15

solution was obtained. After that, the required amount of $\mathrm{HCl}$ was added, and the solution was stirred for another $2 \mathrm{~h}$. In the next stage of procedure $9 \mathrm{~g}$ of tetraethyl orthosilicate, the required amount of aluminium chloride $(0.216 \mathrm{~g})$ was added, and the resulting mixture was continuously stirred at $313 \mathrm{~K}$ for $24 \mathrm{~h}$. The ageing and calcination processes were the same as for SBA- 15 .

\section{Porosity determination}

Nitrogen adsorption/desorption isotherms at $77 \mathrm{~K}$ were obtained volumetrically using ASAP 2020 analyzer (Micromeritics Corp., Norcross, GA, USA). Before the experiment, the adsorbents were degassed $\left(10^{-2} \mathrm{~mm} \mathrm{Hg}\right)$ at $423 \mathrm{~K}$.

The linear BET plots of adsorption data were used to evaluate the BET specific surface area, $\mathrm{S}_{\mathrm{BET}}$. The adsorption/ desorption cumulative pore volume was estimated at the relative pressure $p / p_{0}=0.98$ [13]. The t-plot method was used with the macroporous silica gel LiChrospher Si-1000 as a reference non-porous adsorbent [14] to obtain the values of the external (macropore) surface area $S_{\text {ext }}$, micropore area $\mathrm{S}_{\text {micro }}$ and also for micropore volume $\mathrm{V}_{\text {micro }}$. The calculations of pore size distributions (PSD) followed the Barrett, Joyner and Halenda $(\mathrm{BJH})$ procedure [13]. The adsorption and desorption mesopore diameters were estimated from the PSD maxima. The Horvath-Kawazoe distribution for cylindrical pores model was applied at the relative pressure $p / p_{0}=0.51$ to calculate the median micropore width (Fig. 2).

Results of the porosity measurements for both, Al-SBA-15 vs. SBA-15, are shown in Figs. 1a, b and 2. All the parameters obtained from sorption measurements are given in Table 1.

The isotherms (Fig. 1a) are stepwise with type IV of isotherms with $\mathrm{H} 1$ type of hysteresis loop characteristic for mesoporous materials. The both curves are similar, but the starting point of the hysteresis loop shifts towards lower $p /$ $p_{0}$ values for Al-SBA-15 sample. It is a reflection of the lowering of mesopore diameters caused by the introduction of aluminium into the silica structure. An increase of pore volume and $83 \%$ increase of $\mathrm{S}_{\mathrm{BET}}$ for Al-SBA-15 as compared to pure SBA-15 are observed; it is often found for samples when aluminium was introduced by direct synthesis [15]. The PSD (Fig. 1b) was calculated from the Kelvin equation and is presented as BJH plot. On the PSD desorption curve of the Al-SBA-15 sample, a small peak is observed in the range of 30-37 $\AA$ besides the main intense peak. This may correspond to the larger irregular pores in the aluminium-rich extra-framework species coexisted with the Al-SBA-15 pore network.

To investigate the microporosity of the samples, the differential pore volumes vs. pore widths dependences are shown in Fig. 2. The median micropore widths were determined from the inflection points of these curves (Fig. 2). 


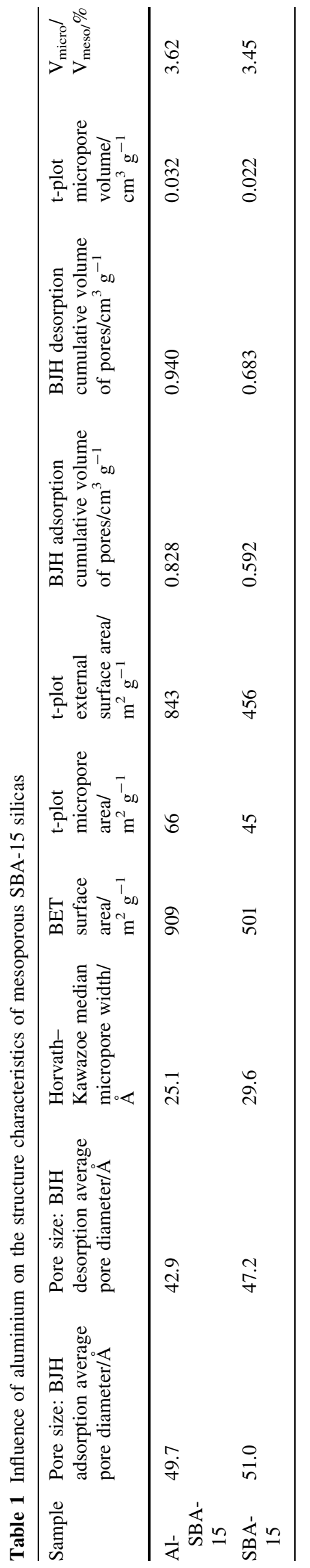

Pore characterization

\section{Small-angle X-ray scattering}

The X-ray diffraction measurements of the synthesized matrices were conducted at the room temperature on the Empyrean powder diffractometer made by Panalytical Co., using a monochromatized $\mathrm{Cu} \quad \mathrm{K}_{\alpha}$ radiation $(\lambda=1.5405980 \AA, P=1.8 \mathrm{~kW})$ in the angular ranging from 0.05 to $5^{\circ}(2 \theta)$ with a step of $0.01^{\circ}$ and with a scanning rate of $0.003^{\circ} \mathrm{s}^{-1}$.

Powder XRD pattern was also collected for SBA-15 before calcination in order to determine the as-synthesized SBA-15 structure.

In Fig. 3a, the XRD patterns of Al-SBA-15 and SBA15 are presented. As followed from Fig. 3a, these patterns consist of three well-resolved peaks at (100), (110) and (200) Bragg reflections, similar to those observed for the as-synthesized SBA-15 (Fig. 3b), indicative of the existence of P6 mm hexagonal symmetry in the materials. The lattice constants calculated from the equation $a=2 d_{100} / \sqrt{ } 3$, where $d_{100}$ is an interplanar spacing, for both samples are same and equal to $114.7 \AA$. Therefore, the $\mathrm{d}$ spacing did not show any changes after $\mathrm{Al}$ introduction. In the case of non-calcined SBA-15 (Fig. 3b), the peaks are much sharper and the lattice parameter increases to $a=124.4 \AA$. The calcination of SBA-15 does not affect its XRD pattern except for increasing the signal intensity. The pore wall thickness $\mathrm{w}$ of the AlSBA-15 sample, calculated from the relation $w=a-$ $\Phi_{\mathrm{BJH}}$, where $\Phi_{\mathrm{BJH}}$ is a pore diameter obtained from adsorption branch of the isotherm, is equal to $65.1 \AA$ and is slightly larger than for pure SBA-15 (63.7 $⿱$ A). It can be caused by a slight distortion of the mesoporous channels or pore narrowing due to the deposition of the Al clusters on the inner pore walls. The SAXS curve for CPG sample (Fig. 3c) shows a broad line with no distinct peaks, which is characteristic for amorphous disordered silica.

\section{Electron microscopy}

TEM micrographs for all samples were obtained with a JEOL JEM-1400 instrument operating at an acceleration voltage $120 \mathrm{kV}$. Its resolution was $0.2 \mathrm{~nm}$. Samples were suspended in methanol and have been subjected an ultrasound sonication for $3 \mathrm{~h}$. One droplet of the suspension was applied to a carbon-coated copper grid and let to dry.

SEM observations were performed on a JEOL JSM$7001 \mathrm{~F}$ scanning electron microscope operating at an acceleration voltage $15 \mathrm{kV}$ and with a resolution $3 \mathrm{~nm}$. Before the irradiation of electron beam, the samples were sputted with a thin gold:palladium coating. 
(a)

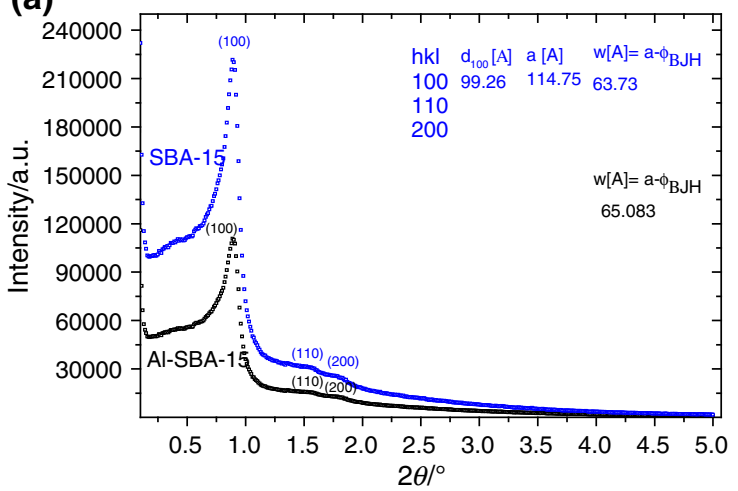

(b)

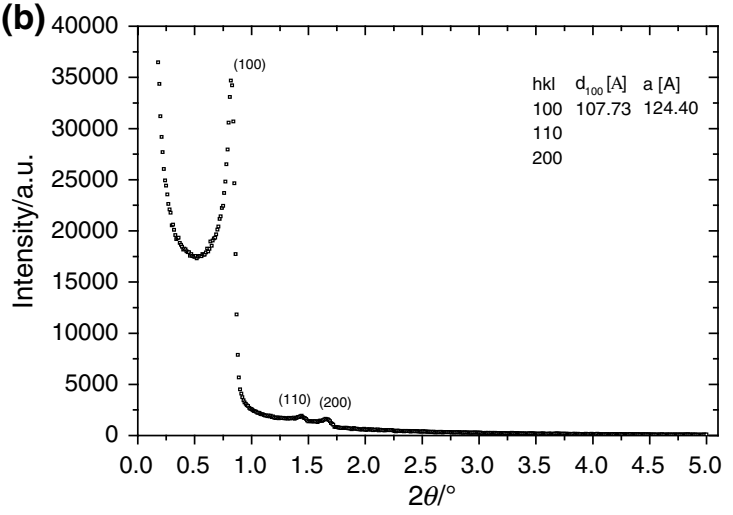

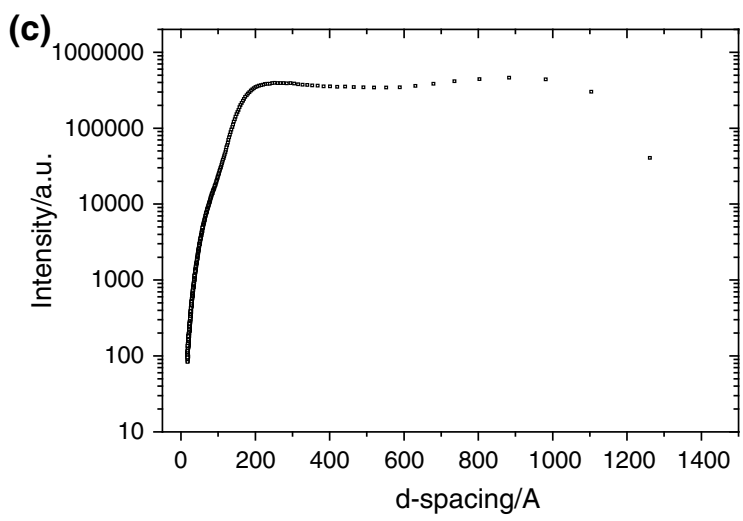

Fig. 3 Small-angle XRD patterns for a the mesoporous Al-SBA-15 and SBA-15 silica, b the as-synthesized SBA-15 and $\mathbf{c}$ CPG glasses
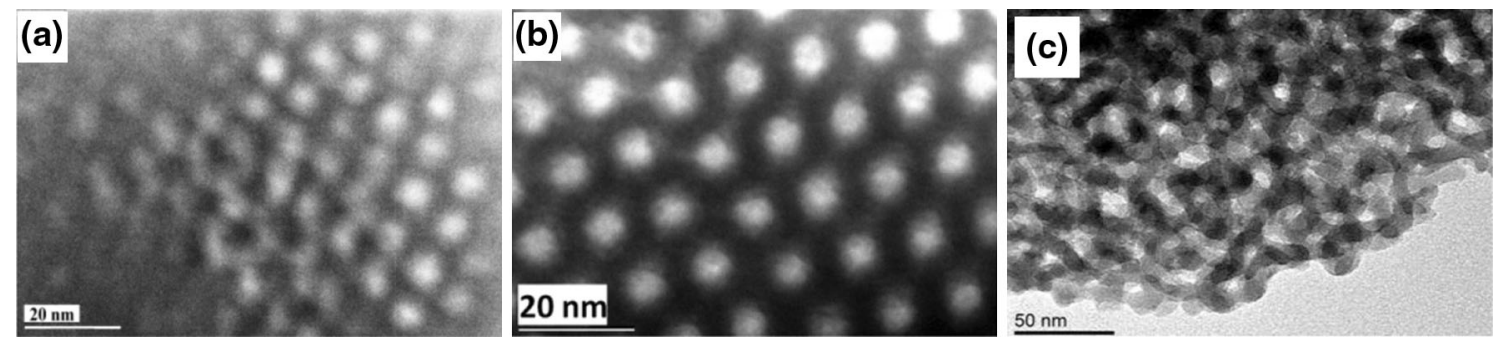

Fig. 4 TEM images of a Al-SBA-15, b SBA-15 and c CPG silica glasses

The chemical composition of Al-SBA-15 sample has been analyzed by the SEM-EDS spectrum at a voltage $15 \mathrm{kV}$. The silicon was selected as an optimization data. $\mathrm{X}$-ray generation occurs as a result of the inelastic scattering process which takes place between incident electrons and the sample, as described elsewhere [16].

Complementary to the XRD data, the TEM images of the Al-SBA-15 (Fig. 4a) and SBA-15 (Fig. 4b) exhibit highly ordered two-dimensional structure of mesopores. It confirms that the hexagonal structure of SBA-15 was preserved after the functionalization. The obtained from TEM images unit cell parameters agree with these from XRD diffraction. Moreover, diameters of Al-SBA-15 mesopores (Fig. 4a) are smaller than for SBA-15 (Fig. 4b), what is in good agreement with the earlier porosity data. The CPG matrix, presented in Fig. 4c, has unordered geometry and does not show any periodic ordering. The morphology of all studied samples is depicted in Fig. 5. The sample of SBA-15 (Fig. 5a) consists of elongated silica fibres with the hexagonal prismatic morphology of the particles, which results from the periodic arrangement of the pores. Similar SEM images of SBA-15 were reported by Kosuge et al. [17]. Figure $5 b$ shows the cross section of non-calcined SBA-15 prismatic particle with a diameter about $1.0 \mu \mathrm{m}$. After calcinations process, the shape of sieves is also preserved. In the case of $\mathrm{Al}$ incorporation (Fig. 5c), the significant change of particle shape is observed. Here, the spherical kind of particles was obtained with diameter ranging from 5 to $12 \mu \mathrm{m}$. 
Fig. 5 SEM images of a SBA15 molecular sieves, $\mathbf{b}$ the assynthesized SBA-15 molecular sieves, c Al-SBA-15 matrix and d CPG glasses
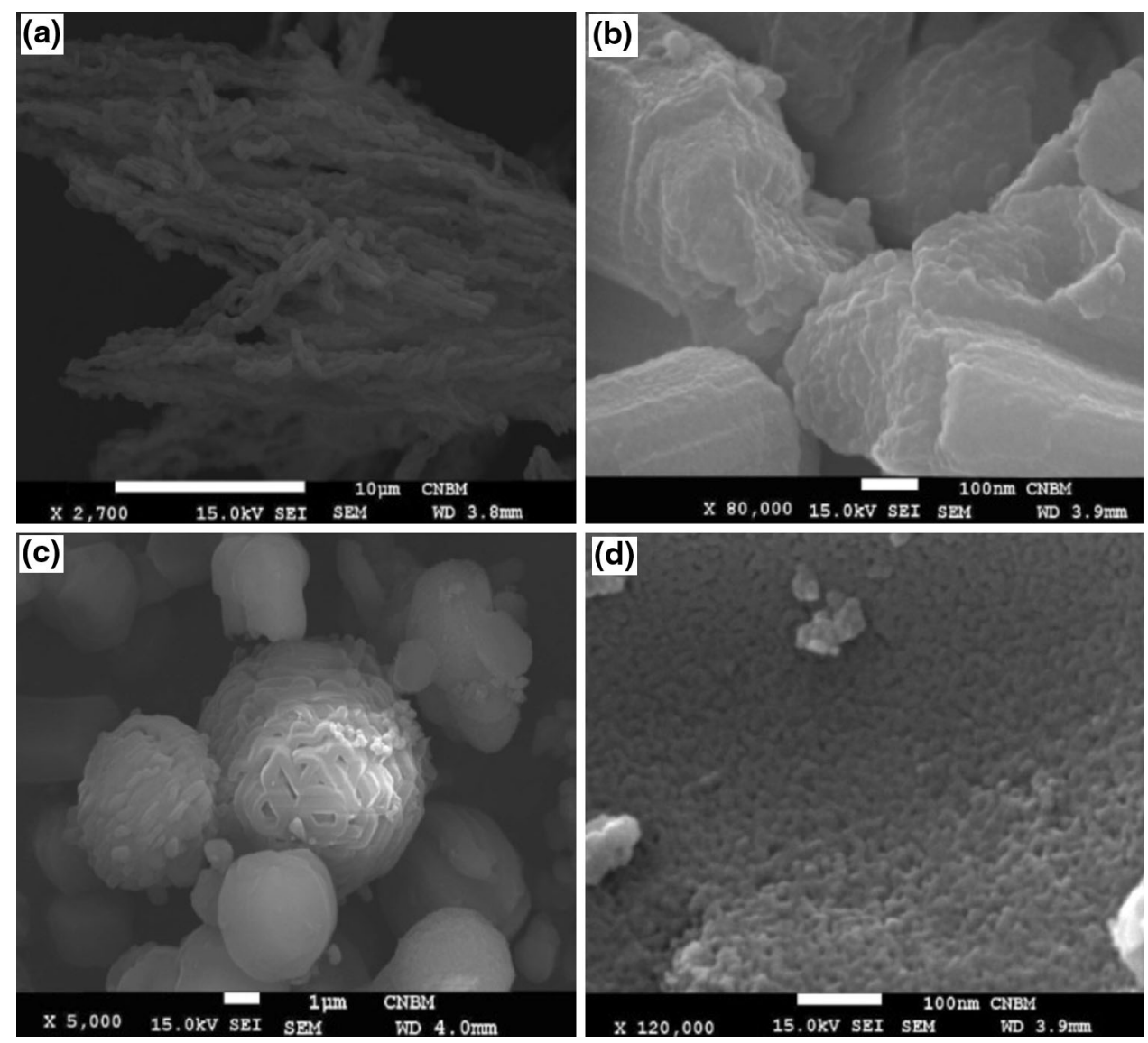

In most cases, these spheres are consisted of parallel layers. CPG silica glasses (Fig. 5d) are the network of interconnected tubular pores, rather than isolated ones.

Using energy-dispersive X-ray spectroscopy for Al-SBA15 sample, the chemical composition and amount of aluminium incorporated in the sieves were obtained. The quantitative analysis of the sample is given in Table 2. As followed from Table 2, the atomic ratio of silicon to aluminium incorporated during synthesis procedure is equal to 517 of atomic percent (529.73 of mass/\%). Figure 6 depicts the EDS spectrum as a plot of X-ray counts vs. energy (in $\mathrm{keV}$ ) indicating the presence of elements such as silicon, oxygen, carbon, chlorine, nitrogen and aluminium as follows from energy peaks correspond to these elements. Generally, they are narrow and readily resolved. The EDS studies show that the synthesized Al-SBA15 matrix contains a very low amount of substituted aluminium, and the influence of heteroatoms and acid sites on adsorptive properties of aluminosilica might be weak.

Differential scanning calorimetry and dielectric relaxation methods

We report experimental studies of the melting transitions for OMCTS confined in SBA-15, Al-SBA-15 and CPG
Table 2 Quantitative results of EDS analysis

\begin{tabular}{lrrr}
\hline Element & Appropriate concentration & Mass/\% & Atomic/\% \\
\hline $\mathrm{C}$ & 131.52 & 263.94 & 46.35 \\
$\mathrm{~N}$ & 1.30 & 9.25 & 1.39 \\
$\mathrm{O}$ & 235.37 & 317.12 & 41.81 \\
$\mathrm{Al}$ & 0.25 & 0.26 & 0.02 \\
$\mathrm{Si}$ & 135.85 & 137.73 & 10.34 \\
$\mathrm{Cl}$ & 1.10 & 1.43 & 0.08 \\
Totals & 729.72 & & \\
\hline
\end{tabular}

$\mathrm{n} / \mathrm{Si} / \mathrm{n} / \mathrm{Al} /=517 /$ atomic $/ \%$

$\mathrm{n} / \mathrm{Si} / / \mathrm{n} / \mathrm{Al} /=529.73 / \mathrm{mass} / \%$

matrices described above, to compare the melting behaviour of OMCTS in pores of the similar pore shape and diameters with the silanoceous and aluminosilanoceous walls and with diversity of the lattice structure, i.e. hexagonal (SBA-15) and amorphous (CPG). Differential scanning calorimetry (DSC) and dielectric spectroscopy (DS) in wide temperature and frequency range were used to determine the melting temperature of confined OMCTS. The porous matrices samples were heated to about $400 \mathrm{~K}$ and kept under vacuum $\left(10^{-4} \mathrm{Tr}\right)$ for a few days to remove the air prior to and during the introduction of the OMCTS. 


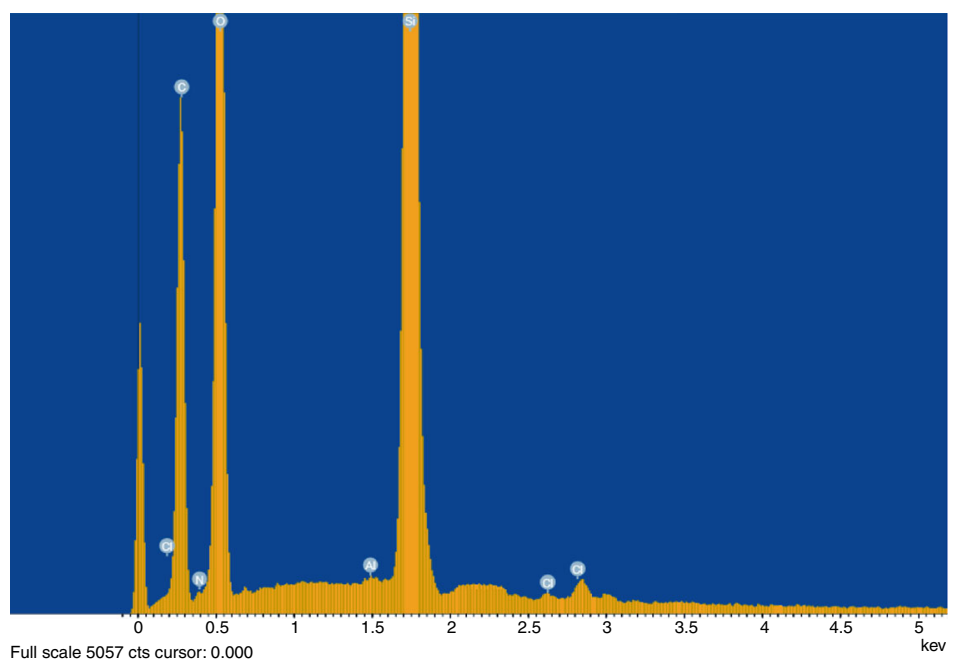

Fig. 6 EDS spectrum of Al-SBA-15 sample

In our experiments, we have used OMCTS of high purity (according to SIGMA catalogue $\geq 99.0 \%$ GC).

A DSC 8000 Perkin Elmer differential scanning calorimeter was used to determine the phase transitions temperatures of the bulk and confined OMCTS by measuring the heat released during phase transitions. All the scans were carried out at heating rates of $5 \mathrm{~K} \mathrm{~min}^{-1}$ in wide range from liquid nitrogen temperature to about $350 \mathrm{~K}$. The temperatures of phase transitions were determined from the position of the peaks of the heat flow during the warming process and were reproducible to within $0.008 \mathrm{~K}$; the enthalpy of bulk OMCTS transitions was obtained from the peak area.

The melting of OMCTS has also been investigated by DS; the method is described elsewhere [18-21]. The electric permittivity is a natural choice of order parameter to study melting of dipolar liquids, because of the large change in the orientational polarizability between the solid and the liquid phases [22]. The complex electric permittivity, $\varepsilon^{*}=\varepsilon^{\prime}+i \varepsilon^{\prime \prime}$ where $\varepsilon^{\prime}=C / C_{0}$ is the real, and $\varepsilon^{\prime \prime}=\tan (\delta), \varepsilon^{\prime}$ is the complex part of the permittivity, was measured at different frequencies and temperatures using a Solartron 1260 impedance gain analyzer, with a parallel plate capacitor made of stainless steel. Here, $C$ is the electric capacitance, $C_{0}$ is the capacitance in the absence of the dielectric medium and $\delta$ is the angle by which the current leads the voltage due to dielectric loss. From the directly measured capacitance, $C$, and the tangent loss $\tan (\delta)$, the values of $\varepsilon^{\prime}$ and $\varepsilon^{\prime \prime}$ were calculated for the known sample geometry. The temperature was controlled to an accuracy of $0.1 \mathrm{~K}$ using a platinum resistor Pt (100) as a sensor and ITC503 Temperature Controller (Oxford Instruments). For an isolated dipole rotating under an oscillating field in a viscous medium, the Debye dispersion relation is derived in terms of classical mechanism:

$\varepsilon^{*}=\varepsilon_{\infty}^{\prime}+\frac{\varepsilon_{s}^{\prime}+\varepsilon^{\prime}}{1+i \omega \tau}$,

where $\omega$ is the frequency of the potential applied, and $\tau$ is the orientational relaxation time of a dipolar molecule. The subscript $\mathrm{s}$ refers to the static permittivity, i.e. the permittivity in the low-frequency limit, when the dipoles have enough time to be in phase with the applied field. The subscript $\infty$ refers to the high-frequency limit of the permittivity and is a measure of the induced component of the

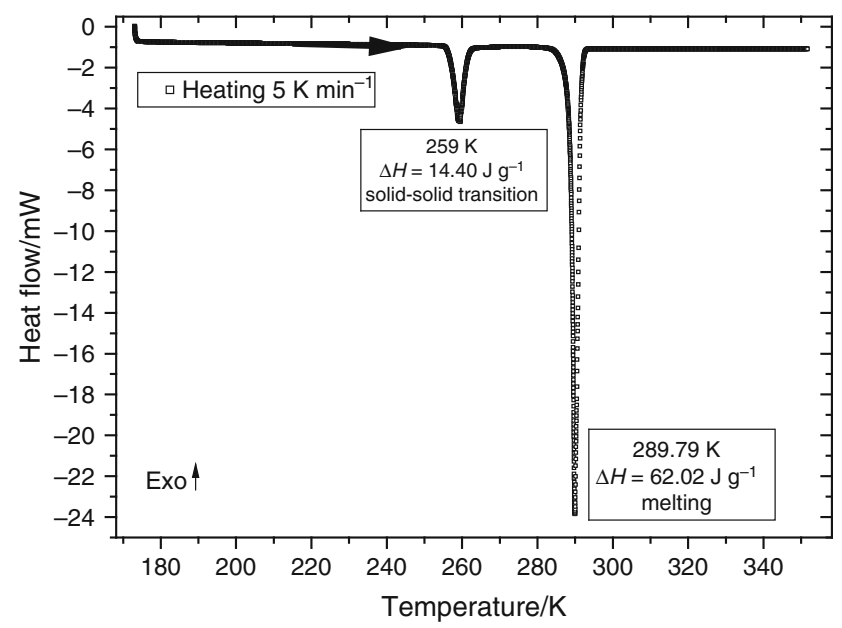

Fig. 7 DSC scan for bulk OMCTS 


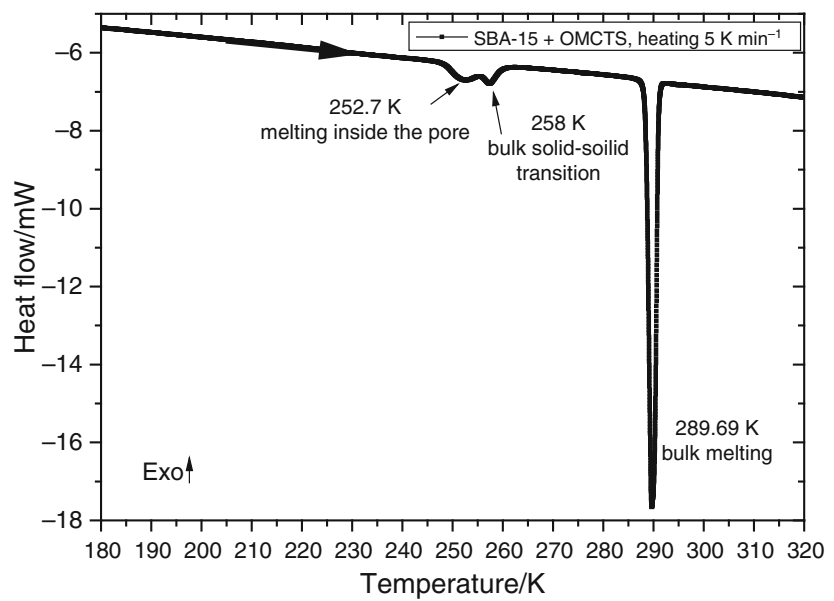

Fig. 8 DSC scan for OMCTS confined in SBA-15 mesopores of diameter $4.9 \mathrm{~nm}$

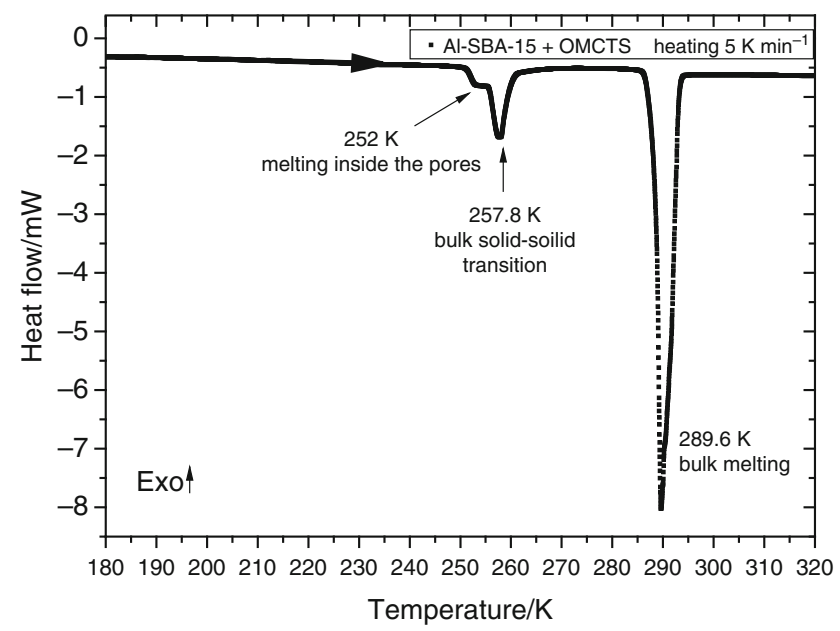

Fig. 9 DSC scan for OMCTS confined in aluminosilica cylindrical Al-SBA-15 mesopores

permittivity. The dielectric relaxation time was calculated by fitting the dispersion spectrum of the complex permittivity near resonance to the Debye model of orientational relaxation.

\section{Results and discussion}

Differential scanning calorimetry results

The melting temperature of bulk OMCTS and the melting behaviour of OMCTS confined in SBA-15 with $4.9 \mathrm{~nm}$ pore size, Al-SBA-15 with mesopores size of $4.6 \mathrm{~nm}$ and CPG of $7.5 \mathrm{~nm}$ pore size were determined using DSC and dielectric methods. Results of the DSC measurements for bulk OMCTS during the heating process as a function of $T$ are shown in Fig. 7. A DSC curve for bulk OMCTS is

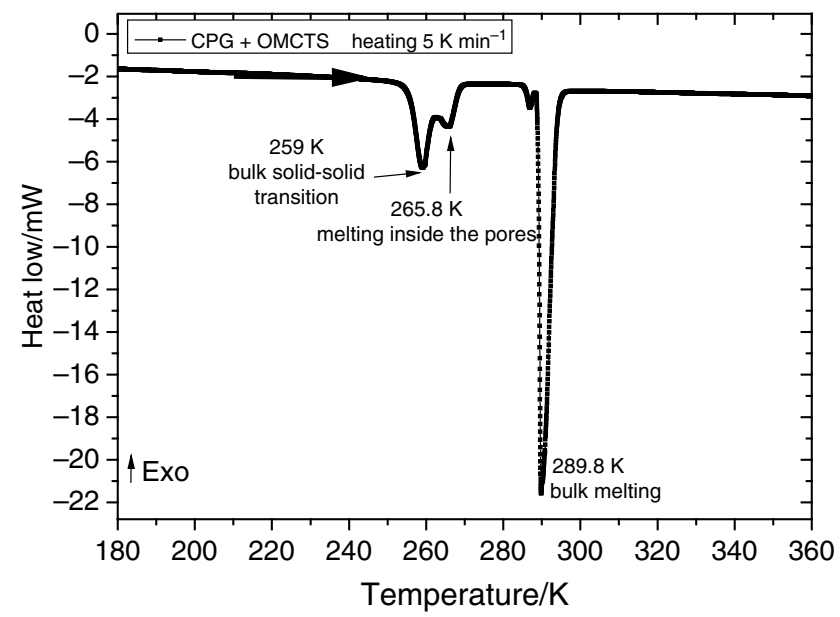

Fig. 10 DSC scan for OMCTS placed in CPG silica glasses of mean diameter $7.5 \mathrm{~nm}$

presented for heating at a temperature scanning rate of $5 \mathrm{~K} \mathrm{~min}^{-1}$. The phase transitions temperatures were determined from the position of the peaks of the heat flow signals on heating and were reproducible to within $0.5 \mathrm{~K}$. The large endothermic peak at $T=289.8 \mathrm{~K}$ corresponds to the melting of the OMCTS, and the smaller peak at $T=259 \mathrm{~K}$ indicates structural change in solid phase [23]. The enthalpy of phase transitions, calculated on the basis of the values of peaks area of DSC scans, for melting transition is almost 4.5 times higher than for the solid-solid transition (Fig. 7). It can indicate the existence of solidsolid transitions in OMCTS observed at temperature below the melting point.

In Fig. 8, the DSC scan is presented for OMCTS confined in SBA-15 with mesopores of diameter of $4.9 \mathrm{~nm}$. Since the samples studied are a suspension of OMCTSfilled silica sieves in pure OMCTS, the signals contain both contributions of bulk and confined OMCTS. The large endothermic peak at $289.7 \mathrm{~K}$ corresponds to the melting of the bulk OMCTS; the second peak at $258 \mathrm{~K}$ corresponds to bulk structural phase transition in the solid state. In addition, a next peak at $252.7 \mathrm{~K}$ is observed, which can correspond to the melting of OMCTS in SBA-15. Then, the melting temperature in pores is shifted towards lower temperatures relatively to the melting of bulk OMCTS by about $\Delta T=T_{\mathrm{m} \text {,pore }}-T_{\mathrm{m} \text {,bulk }}=-37 \mathrm{~K}$. As followed from Fig. 8, the temperature of solid-solid transition for confined system is not observed at DSC scan. It can suggest that the solid-solid transition not appears inside the pores. Such a result is confirmed also by dielectric relaxation method. The depression of the melting point observed in this system can be explained by relatively small value of the wetting parameter $\alpha$ in pores because of the small fluidwall interactions and the roughness of the pore walls [24]. 
In Fig. 9, the DSC scan for OMCTS confined in aluminosilica matrix having mesopores of pore size of $4.6 \mathrm{~nm}$ during the heating process is presented. As followed from Fig. 9, the result is similar to this obtained for OMCTS confined in SBA-15 matrix. We observe the peaks related with the solid-solid and melting transition of the bulk OMCTS; the melting temperature of OMCTS in Al-SBA15 is observed at $252 \mathrm{~K}$. The value is only $0.7 \mathrm{~K}$ lower than the melting point of OMCTS placed in SBA-15 i.e. $T_{\mathrm{m} \text {,pore }}=252.7 \mathrm{~K}$. The pore size and the ratio between micropores volume and mesopores volume $\mathrm{V}_{\text {micro }} / \mathrm{V}_{\text {meso }}$ (see Table 1) of both matrices are comparable, and the amount of incorporated aluminium into silica wall is small. It caused that there is no significant difference $\left(\Delta T_{\mathrm{m} \text {,pore }}=0.7 \mathrm{~K}\right)$ between melting temperatures of OMCTS in SBA-15 and Al-SBA-15. As followed from DSC scan presented in Fig. 9, the solid-solid transition in confined system is not observed at this system.

In order to verify whether and how the kind of structure and of pore wall affects on adsorptive properties of the matrix, we have performed measurements for OMCTS confined into CPG silica glasses of mean diameter $7.5 \mathrm{~nm}$, using DSC method.

In Fig. 10, the DSC scan for OMCTS confined in CPG during the heating process is presented. The large peak at $289.8 \mathrm{~K}$ corresponds to the melting of the bulk OMCTS, and the temperature of solid-solid transition of the OMCTS bulk is shown as an endothermic peak at $258 \mathrm{~K}$. The melting inside the pores can be related with the phase transition observed as a DSC peak at $265.8 \mathrm{~K}$. The shift of the melting point of OMCTS in CPG pores relatively to the bulk is equal to $\Delta T=-24 \mathrm{~K}$ and is smaller than in SBA15 and Al-SBA-15. Moreover, we have not observed the solid-solid transition of the confined OMCTS. The pore size of CPG is equal to $7.5 \mathrm{~nm}$. Many experimental results and some theoretical data $[25,26,32]$ show that in case of cylindrical pores of diameter larger than $7 \mathrm{~nm}$, the shift in the melting temperature is linearly related to the inverse pore width according to the Gibbs-Thomson equation of thermodynamics $[27,28,33]$ :

$\Delta T_{\mathrm{m}}=T_{\mathrm{m}, \text { pore }}-T_{\mathrm{m}, \text { bulk }}=-2 \frac{\left(\gamma_{\mathrm{ws}}-\gamma_{\mathrm{wf}}\right) v}{H \gamma_{\mathrm{m}, \text { bulk }}}$,

where $\gamma_{\mathrm{ws}}$ and $\gamma_{\mathrm{wf}}$ are the wall-solid and wall-fluid surface tensions (here solid refers to the solid phase of the adsorbate), $v$ is the molar volume of the liquid phase and $\lambda_{\mathrm{m} \text {,bulk }}$ is the bulk latent heat of melting. This equation fails for pores much smaller than about $7 \mathrm{~nm}$ in width for the systems considered. Such breakdown for narrow pores is expected, since the equation rests on macroscopic concepts such as surface tension (implying a well-defined interface between two bulk-like phases) and fails to account for the inhomogeneity of the confined phase [19]. On the basis of Eq. (2), we can expect increase of melting temperature of OMCTS in CPG relatively to the melting point of OMCTS in SBA-15 and Al-SBA-15 with pore size about $4 \mathrm{~nm}$.

\section{Dielectric relaxation spectroscopy results}

Similar results were obtained using DRS method. Results of the measurements of $C$ for bulk OMCTS during the heating process as a function of $T$ at a frequency of $200 \mathrm{kHz}$ is shown in Fig. 11a. There is a sharp increase in $C$ at $T=290 \mathrm{~K}$, the melting point of the pure substance, due to the contribution to the orientational polarization in the liquid state from the permanent dipoles. At the temperature $T=259 \mathrm{~K}$, the second sharp increase of $C$ is observed, which can correspond to the structural transition of solid phases of OMCTS as it appears below the melting point of OMCTS. Analysis of the Cole-Cole representations of the complex capacity [5] for solid OMCTS has shown that the relaxation observed can be described by a symmetric ColeCole distribution of relaxation times. Examples of the experimental points and fitted curves are given in Fig. 11b-e for the solid OMCTS at 196 and $281 \mathrm{~K}$, and for the OMCTS liquid phase at $294 \mathrm{~K}$ (Fig. 11f, g).

From the plot of $\varepsilon^{\prime}$ and $\varepsilon^{\prime \prime}$ vs. $\log \omega$ (Fig. 11c, e, g), the relaxation time can be calculated as the inverse of the frequency $\omega$ corresponding to a saddle point of the $\varepsilon$ plot or a maximum of the $\varepsilon^{\prime \prime}$ plot. An alternative graphical representation of the Debye dispersion equations is the Cole-Cole diagram in the complex $\varepsilon^{*}$ plane (Fig. 11b, d, f). Each relaxation mechanism is reflected as a semicircle in the Cole-Cole diagram. From the plot of $\varepsilon^{\prime \prime}$ vs. $\varepsilon^{\prime}$, the value of $\tau$ is given as the inverse of the frequency at which $\varepsilon^{\prime \prime}$ goes through a maximum.

Figure $11 \mathrm{~h}$ presents the variation of the relaxation time with temperature for bulk OMCTS obtained from fitting Eq. (1) to the dispersion spectrum. In the lower temperature range until about $259 \mathrm{~K}$, a single relaxation time of the order of $10^{-3} \mathrm{~s}$, the value typical for the solid crystal form, is observed. In the temperature ranging from $259 \mathrm{~K}$ to about $290 \mathrm{~K}$, we observe the strong temperature dependence of the branch of relaxation time, which increase with increasing temperature until OMCTS melting point at $290 \mathrm{~K}$. The strong temperature dependence of $\tau$ in the temperature range of 259-290 K can suggest the existence of the plastic form of solid OMCTS at this temperature range; at $259 \mathrm{~K}$, the transition to different crystal form of OMCTS is observed. The phase transition typical for plastic crystals, from the phases where the molecules possess unusual freedom of movement, is typical for "globular" molecules with the remarkably small entropy of fusion, to the high crystallographic symmetry form [29]. Our preliminary results 
(a)

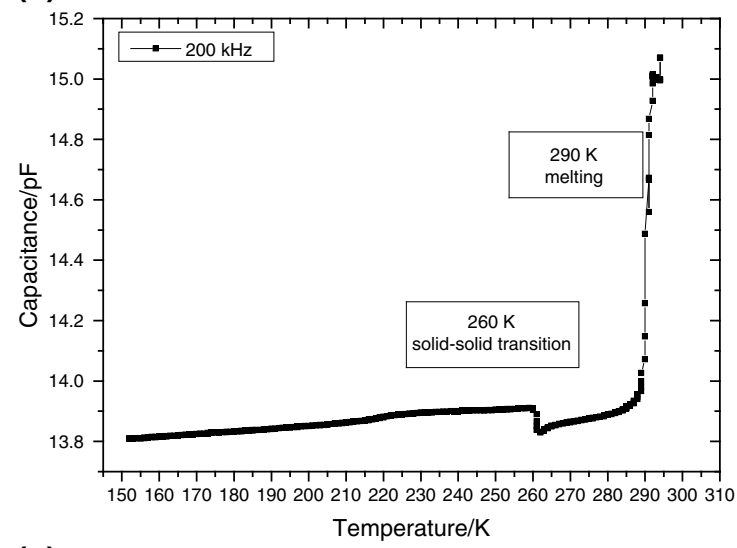

(c)

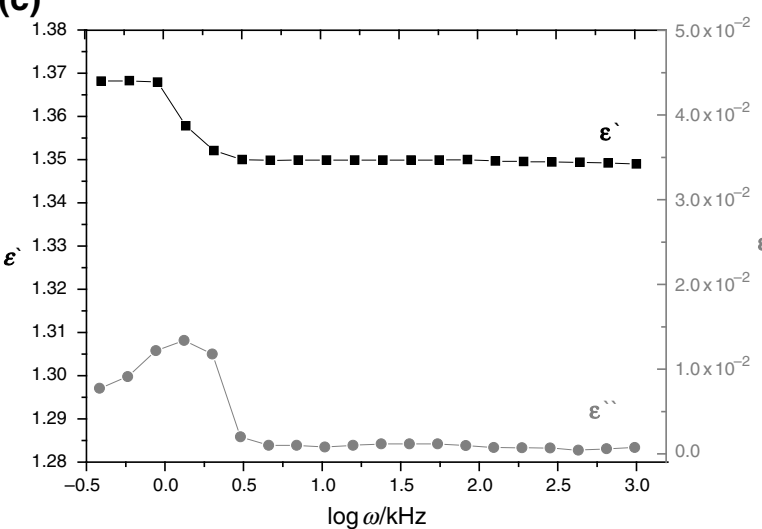

(e)

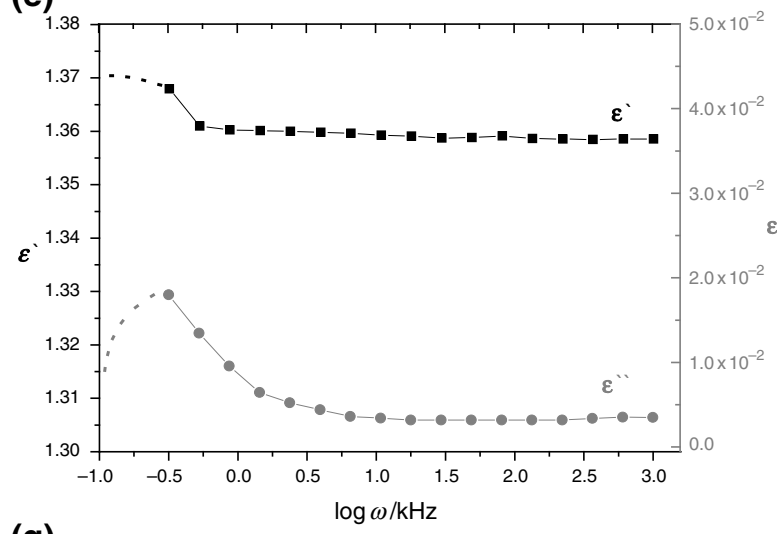

(g)

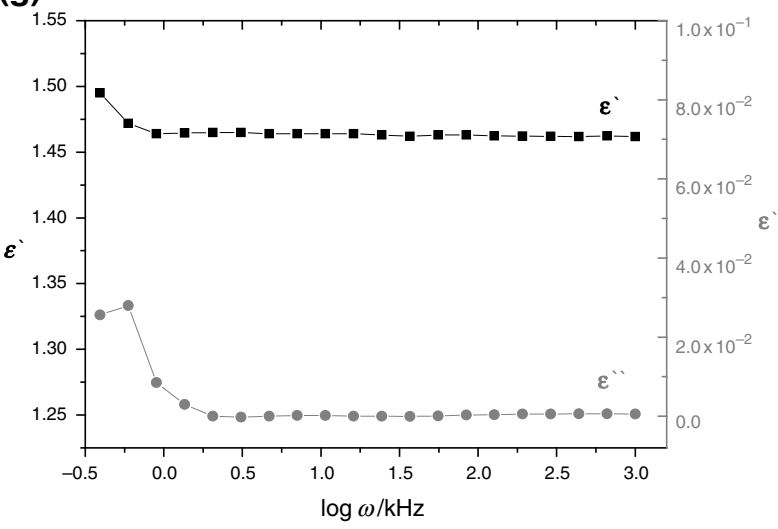

(b)

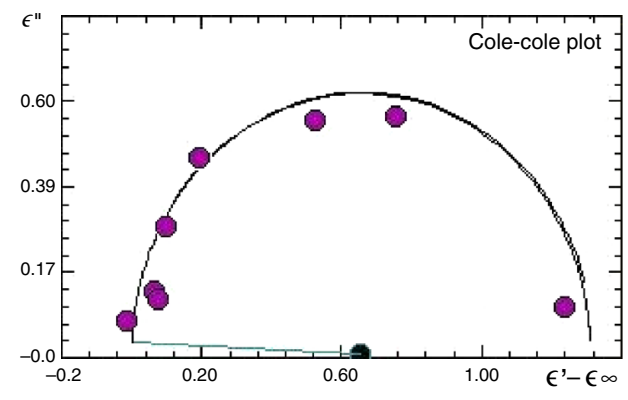

(d)

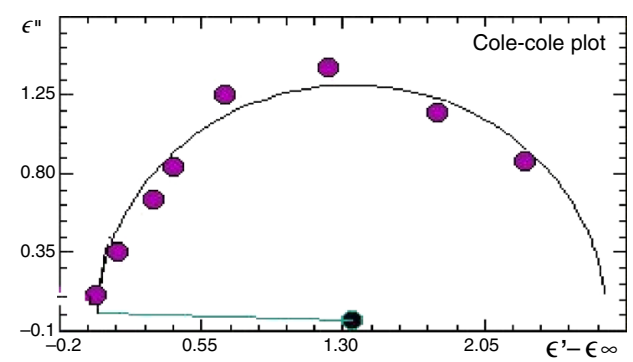

(f)

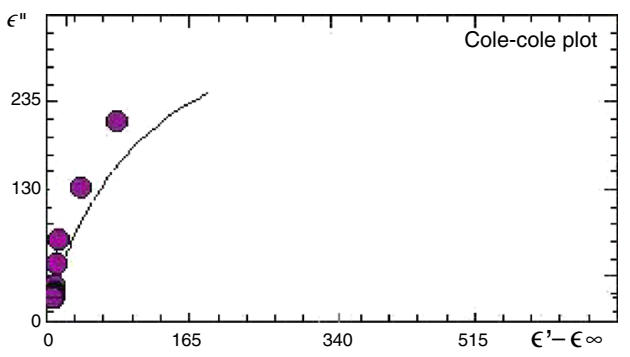

(h)

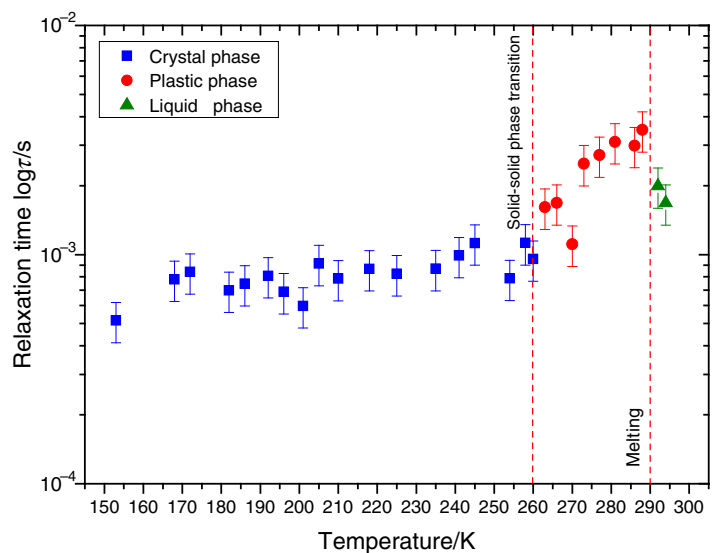


4Fig. 11 a Capacitance $C$ versus temperature for bulk OMCTS, b a Cole-Cole diagram for bulk OMCTS at $196 \mathrm{~K}$, c spectrum plot for OMCTS at $196 \mathrm{~K}$, d a Cole-Cole diagram for bulk OMCTS at $281 \mathrm{~K}$, e spectrum plot for OMCTS at $281 \mathrm{~K}$, f a Cole-Cole diagram for melted OMCTS at $294 \mathrm{~K}$, g spectrum plot for OMCTS at $294 \mathrm{~K}$ and $\mathbf{h}$ dielectric relaxation time $\tau$ versus temperature for bulk OMCTS

obtained using WAXS methods for OMCTS shown the existence of high crystallographic symmetry form of OM$\mathrm{CTS}$ at the temperature range below $259 \mathrm{~K}$ [to be published]. At temperatures above the OMCTS melting point of $290 \mathrm{~K}$, a change of the value of the relaxation time observed is related to the transition to the liquid phase. The corresponding diagrams are shown in Fig. 11e and f. In the presence of dipolar constituents, one or more absorption regions are present, not all of them necessarily associated with the dipolar dispersion. At the lowest frequencies (especially about hundreds $\mathrm{Hz}$ ), a large $\varepsilon^{\prime \prime}$ value arises from the conductivity of the medium, and interfacial (MaxwellWagner) polarization is observed. For OMCTS a homogeneous medium whose conductivity is of the order of $10^{-9} \Omega^{-1} \mathrm{~m}^{-1}$, the absorption region observed for the lower frequencies is related to the conductivity of the medium. The Joule heat arising from the conductivity contributes to a loss factor $\varepsilon^{\prime \prime}$ (conductance) so that the value at low frequency is $\varepsilon_{\text {total }}^{\prime \prime}=\varepsilon_{\text {dielectric }}^{\prime \prime}+\varepsilon_{\text {conductance, }}^{\prime \prime}$ and the system reveals the energy loss in processes other than dielectric relaxation [21]. In Fig. 11g, the component of the relaxation time of the order of $10^{-3} \mathrm{~s}$ characterizes the process of absorption related to the conductivity of the medium. This branch, strongly dependent on temperature, is characteristic for the liquid phase and is a good indicator of the appearance of the phase.

In Figs. 12 and 13, the capacity $C$ is presented as a function of temperature for OMCTS confined in SBA-15

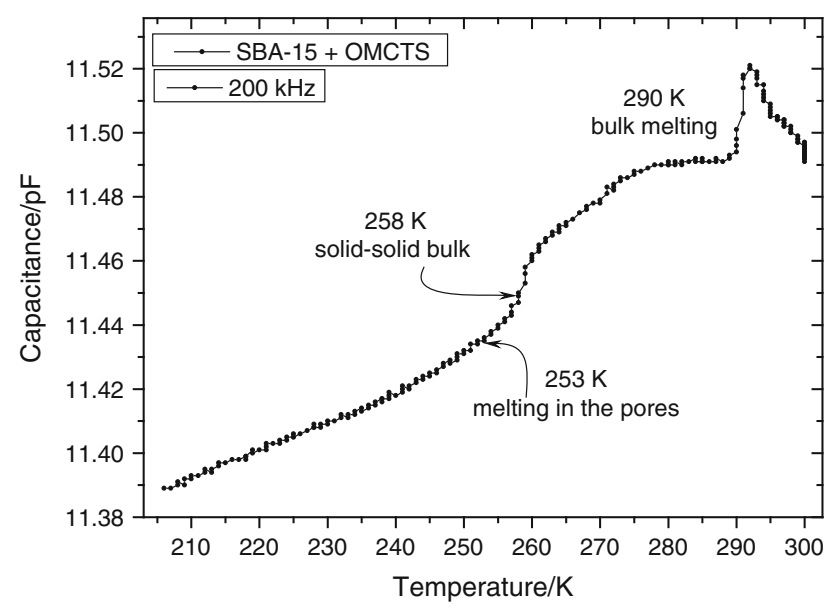

Fig. 12 Capacitance $C$ versus temperature for OMCTS confined in SBA-15 mesopores of diameter $4.9 \mathrm{~nm}$

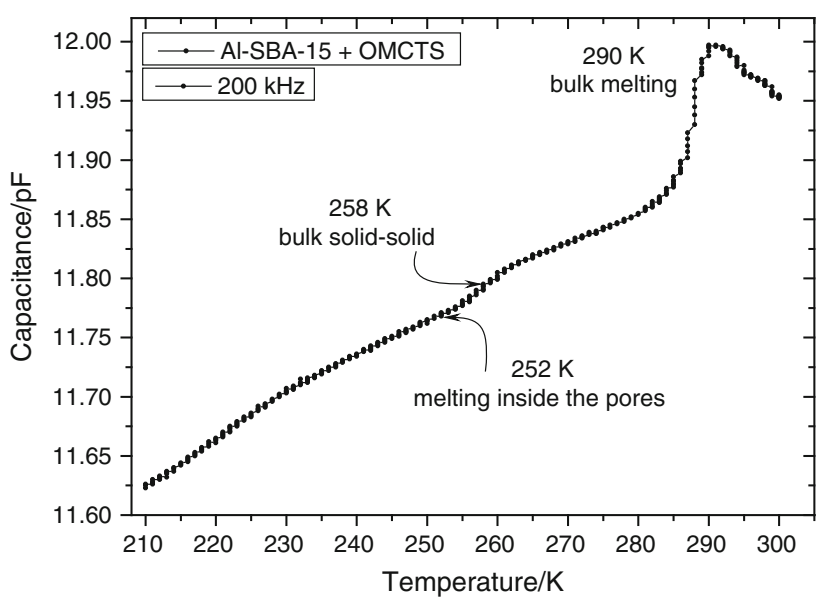

Fig. 13 Capacitance $C$ versus temperature for OMCTS confined inside the Al-SBA-15 mesopores of $4.6 \mathrm{~nm}$ diameter

mesopores of diameter of $4.9 \mathrm{~nm}$ and in Al-SBA-15 mesopores of diameter of $4.6 \mathrm{~nm}$, respectively. As followed from Figs. 12 and 13, the bulk melting of OMCTS shown as a sharp decrease of $C(T)$ functions was found in the temperature $290 \mathrm{~K}$ for both systems. The solid-solid transition of bulk OMCTS was observed at the temperature equal $258 \mathrm{~K}$, what is in good agreement with the DSC data. The pore melting is attributed to a sharp increase of the $C(T)$ function at $253 \mathrm{~K}$ in SBA- 15 and at $252 \mathrm{~K}$ in $\mathrm{Al}-$ SBA-15. The depression of the pore melting point relative to the bulk for OMCTS confined in Al-SBA-15 is equal to $\Delta T=-38 \mathrm{~K}$ and is about $1 \mathrm{~K}$ greater than for SBA-15.

For OMCTS confined in Al-SBA-15, the behaviour of the relaxation times vs. temperature has a similar character to that shown in Fig. 12. For both systems, we can conclude that below of the melting transitions in pores at $252 \mathrm{~K}$ (in the case of OMCTS in Al-SBA-15) and $253 \mathrm{~K}$ (for OMCTS in SBA-15), we observe the existence of high crystallographic symmetry form which transform to the liquid phase above the melting points in pores. As followed from our DSC and DRS measurements, we did not observe the plastic form of OMCTS inside the pores.

Figure 14 presents the variation of the relaxation time $\tau$ with temperature $T$ for OMCTS confined in SBA-15 matrix, obtained from fitting Eq. (1) to the dispersion spectrum. As followed from Fig. 14 at the temperature range below $253 \mathrm{~K}$, the system shows a single relaxation time lower than $10^{-3} \mathrm{~s}$, the branch typical for the solid crystal form of OMCTS (Fig. 11h). It can suggest that the high crystallographic symmetry form exists in the OMCTS bulk and also inside the pores below the pore melting point. Above the temperature of the pore melting point $253 \mathrm{~K}$, we can observe an additional component of relaxation time, which can arise from the conductivity of the liquid inside the pores and indicate the existence of the liquid OMCTS 


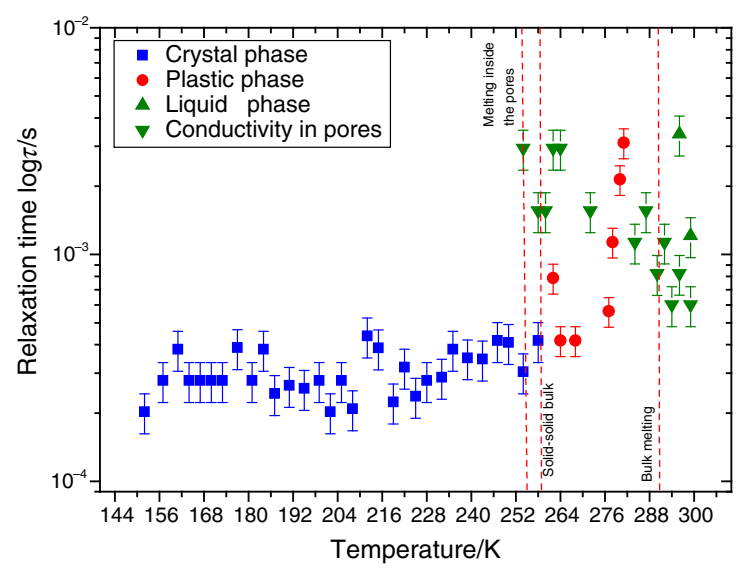

(a)

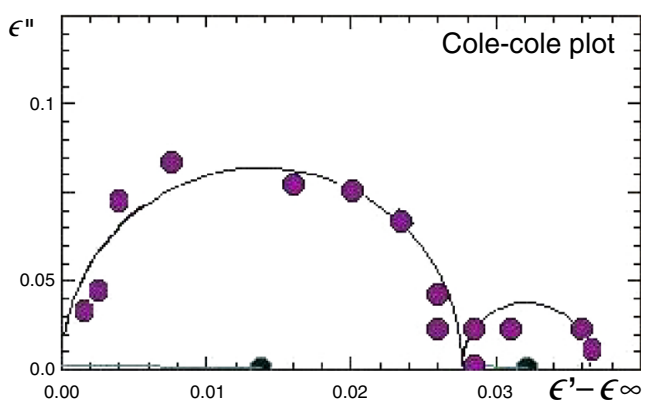

(b)

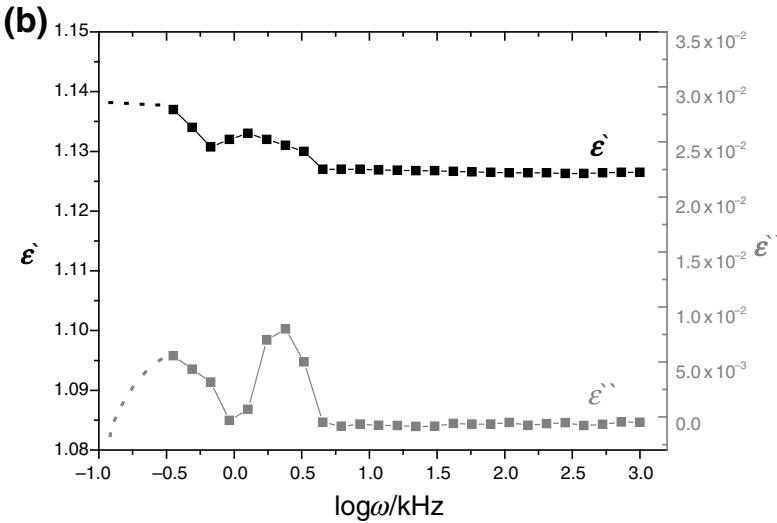

Fig. 14 Dielectric relaxation time $\tau$ versus temperature for OMCTS confined in SBA-15 mesopores of diameter $4.9 \mathrm{~nm}$; a A Cole-Cole diagram at $264 \mathrm{~K}$, b spectrum plot for OMCTS at $264 \mathrm{~K}$

in the sample in this temperature range. In the temperature range $258-290 \mathrm{~K}$, the two branches of the relaxation time are observed: one of the order of $10^{-2}-10^{-3} \mathrm{~s}$ corresponds to the conductivity inside the pores and the second of the order of $10^{-2}-10^{-4} \mathrm{~s}$ which is typical for the plastic form of bulk OMCTS. In Fig. 14a, an example of analysis of the Cole-Cole representation of the complex capacity for OMCTS in SBA-15 at $264 \mathrm{~K}$ is shown; Fig. 14b presents the spectrum plot $\left(\varepsilon^{\prime}\right.$ and $\varepsilon^{\prime \prime}$ vs. $\left.\log \omega\right)$ for this system at $264 \mathrm{~K}$. This spectrum represents two relaxation mechanisms, corresponding to the two semi-circles in the ColeCole diagram, characterized by relaxation times of the order of $5 \times 10^{-4}$ and $10^{-3} \mathrm{~s}$, respectively. At temperatures above the bulk OMCTS melting point of $290 \mathrm{~K}$ (Fig. 14), the branch of $\tau$ related with the conductivity inside the pores and the liquid phase of bulk OMCTS is observed.

In Fig. 15, the dependence of $\Delta T$ vs. $H^{-1}$ for all systems studied is presented. We can observe that for smaller pore sizes, the depression of $\Delta T$ increases; for OMCTS in CPG, the shift in the melting temperature is linearly related to the inverse pore width according to the Gibbs-Thomson equation of thermodynamics. For the smaller pores, this linear

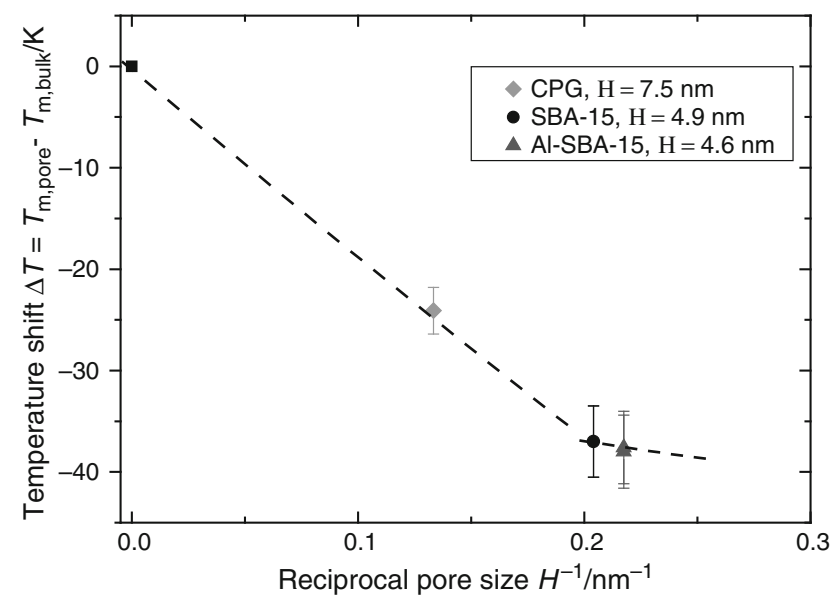

Fig. 15 The temperature shifts versus the reciprocal pore size for OMCTS placed in CPG silica glasses, SBA-15 and Al-SBA-15

behaviour is not observed. In the case of cylindrical silicabased pores, it was shown that a homogeneous crystalline confined phase results only for cylindrical pores with average diameters larger than about $12 \sigma_{\mathrm{ff}}$. For cylindrical pores with lower diameter, the confined phase at low temperature 
is an inhomogeneous phase with partially crystalline domains interspersed with amorphous regions [19].

For OMCTS in SBA-15 and Al-SBA-15 with pore size about $4 \mathrm{~nm}$, we can expect that inside the pores below the melting temperature rather inhomogeneous phase will be formed, and at the solid phase, the crystal form can be partially deformed [30, 31]. In such a case, the solid-solid transition observed in OMCTS bulk does not occur in the pores. As followed from Fig. 15, the dependence of $\Delta T$ vs. $H^{-1}$ observed for the systems studied suggests that the differences in melting behaviour between CPG, SBA-15 and Al-SBA-15 observed in our experiments are primarily due to the difference in pore size. The influence of the surface roughness and chemical heterogeneity for the ordered porous systems e.g. cylindrical SBA-15 and AlSBA-15 is rather weak.

\section{Conclusions and future work}

We have investigated the thermal and dielectric properties of the OMCTS liquid under confinement conditions in silica and aluminosilica glasses and CPG. The obtained matrices by us have the hexagonal symmetry and the following mesopores diameters: SBA-15-4.9 $\mathrm{nm}$ and AlSBA-15-4.6 nm. Studied CPG glasses are amorphous disordered silica glass with a mean pore diameter $7.5 \mathrm{~nm}$. The substitution of heteroatoms with valence lower than silica (as aluminium) creates negative charges in the silica framework, which are compensated by protons, generating strong Broensted acidity of bridging $\mathrm{Si}-(\mathrm{OH})-\mathrm{Al}$ sites in SBA-15. Our nitrogen sorption studies of Al-SBA-15 material have shown a decrease of mesopore size from $4.9 \mathrm{~nm}$ for pure SBA-15 to $4.6 \mathrm{~nm}$ due to the deposition of the $\mathrm{Al}$ clusters on the inner pore walls and an increase of the total pore volume and $\mathrm{S}_{\mathrm{BET}}$ surface area (83\%), thereby improving textural and adsorptive properties of SBA-15 matrix. The characterizations using SAXS and TEM methods have confirmed the preservation of hexagonal P6 mm structure after Al functionalization, showing that aluminium can improve the acidity and the interaction between the host and the guests (OMCTS) molecules as well. As followed from EDS analysis, the aluminium content in silica framework is equal to 0.26 mass\%, resulting $\mathrm{n}(\mathrm{Si}) / \mathrm{n}(\mathrm{Al})$ ratio equal to 529 .

Analysis of the thermal properties of OMCTS bulk by dielectric and DSC methods shows that OMCTS in the solid state exhibits the phase transition typical for the transition from high crystallographic symmetry form to the plastic crystal form. From our experiments, we can suggest that for confined OMCTS, the transition from high crystallographic symmetry form to the plastic form is not observed.
We have observed depression of the melting point for OMCTS confined in the cylindrical pores of both: silica and aluminosilica matrices, as well as for disordered CPG matrix. The depression is larger for hexagonal matrix than for CPG due to the smaller pore diameters of this matrix. The thermal response of OMCTS confined inside silica and aluminosilica matrix shows the decrease of melting temperature in Al-SBA-15 relatively to the SBA-15. The EDS results have shown that the amount of incorporated aluminium into the SBA-15 framework is 0.02 ; hence, influences of aluminium heteroatoms and acid sites on adsorptive properties of SBA-15 matrix are not too strong, but we can observe that the difference between the melting points is about $1 \mathrm{~K}$. Moreover, OMCTS molecule with all saturated bonds and the oxygen atoms which are not exposed can interact with silica and aluminosilica walls by weak van der Waals' forces.

The weak differences in melting point depression of OMCTS confined in silica and aluminosilica mesopores are consistent with the results of our estimation of wetting parameter of OMCTS in these pores [24]. The influence of energetics (fluid-fluid and fluid-wall interactions) on the freezing/melting behaviour of the fluids confined in porous materials can be understood in terms of two main parameters, as proposed in our previous work [6]. One is the pore width $H$, the latter is the wettability parameter $a_{\mathrm{w}}$, $\alpha_{\mathrm{w}}=\frac{\rho_{\mathrm{w}} \varepsilon_{\mathrm{fw}} \sigma^{2} f \mathrm{v} \Delta}{\varepsilon_{\mathrm{ff}}}$, which is the ratio of the fluid-wall to the fluid-fluid attractive interaction, where $\Delta$ is the inter-layer spacing in the solid and $\rho_{\mathrm{w}}$ is the number of wall atoms per unit area. Our previous results [3, 6, 30-32] indicate that the critical $\alpha_{\mathrm{w}}$ value for Lennard-Jones fluids in smooth-walled pores is about 1 , and thus for alpha greater than 1 , we have strongly attractive pores-like a wall of graphite, and then occurs an elevation in freezing temperature-while for values of alpha less than 1 , we have weakly attractive pores-like a wall of silica, and then occurs a depression in freezing temperature. Our experimental evaluation of the effective wettability parameter $\alpha_{\mathrm{p}}$ based on the measurements of contact angle of OMCTS inside the silica pores has shown that the effective value of $\alpha$ in silica pores is about $\alpha_{\mathrm{p}}=0.4$ [24], and we can expect the depression of the melting temperature for OMCTS in the studied matrices.

Acknowledgements This work has been partially supported by the Operational Program 'Human Capital'-PO KL 4.1.1, 'Proinnowacyjne kształcenie, kompetentna kadra, absolwenci przyszłości'. We thank for financial support for National Center of Science, Grant No: DEC-2013/09/B/ST4/03711. Financial Support from the National Centre for Research and Development under research Grant No PBSI/A9/13/2012 is also gratefully acknowledged.

Open Access This article is distributed under the terms of the Creative Commons Attribution License which permits any use, distribution, and reproduction in any medium, provided the original author(s) and the source are credited. 


\section{References}

1. Radhakrishnan R, Gubbins KE, Sliwinska-Bartkowiak M. Global phase diagrams for freezing in porous media. J Chem Phys. 2002;116:1147-55.

2. Christenson HK. Confinement effects on freezing and melting. J Phys: Condens Matter. 2001;13:R95-133.

3. Sliwinska-Bartkowiak M, Hung FR, Santiso EE, Coasne B, Dudziak G, Siperstein FR, Gubbins KE. Effect of confinement on freezing of $\mathrm{CCl}_{4}$ in cylindrical pores. Adsorption. 2005;11:391-6.

4. Alba-Simionesco C, Coasne B, Dosseh G, Dudziak G, Gubbins KE, Radhakrishnan R, Sliwinska-Bartkowiak M. Effects of confinement on freezing and melting. J Phys: Condens Matter. 2006;18:R15-68.

5. Sliwinska-Bartkowiak M, Gras J, Sikorski R, Radhakrishnan R, Gelb L, Gubbins KE. Phase transitions in pores: experimental and simulation studies of melting and freezing. Langmuir. 1999;15:6060-9.

6. Radhakrishnan R, Gubbins KE, Sliwinska-Bartkowiak M. Effect of the fluid-wall interaction on freezing of confined fluids: towards the development of a global phase diagrams. J Chem Phys. 2000;112:11048-57.

7. Klein J, Kumacheva E. Simple liquids confined to molecularly thin layers. I. Confinement-induced liquid-to-solid phase transitions. J Chem Phys. 1998;108(16):6996-7009.

8. Horn RG, Israelachvili JN. Direct measurement of structural forces between two surfaces in a nonpolar liquid. J Chem Phys. 1981;75:1400-11.

9. Levent Demirent A, Granick S. Origins of solidification when a simple molecular fluid is confined between two plates. J Chem Phys. 2001;115(3):1498-512.

10. Christenson HG, Israelachvili JN. Temperature dependence of solvation forces. J Chem Phys. 1984;80:4566-7.

11. Bhange P, Bhange DS, Pradhan S, Ramaswamy V. Direct synthesis of well-ordered mesoporous Al-SBA-15 and its correlation with the catalytic activity. Appl Catalys A General. 2011;400:176-84.

12. Joo SH, Ryoo R, Kruk M, Jaroniec M. Evidence for general nature of pore interconnectivity in 2-dimensional hexagonal mesoporous silicas prepared using block copolymer templates. J Phys Chem B. 2002;106:4640-6.

13. Gregg SJ, Sing KSW. Adsorption, surface area and porosity. London: Academic; 1982

14. Jaroniec M, Kruk M, Olivier JP. Standard nitrogen adsorption data for characterization of nanoporous silicas. Langmuir. 1999;15(16):5410-3.

15. Kumaran GM, Garg S, Soni K, Kumar M, Gupta JK, Sharma LD, Rama Rao KS, Murali Dhar G. Synthesis and characterization of acidic properties of Al-SBA-15 materials with varying $\mathrm{Si} / \mathrm{Al}$ ratios. Micro Meso Mater. 2008;114:103-9.

16. Goldstein J. Scanning electron microscopy and X-ray microanalysis. New York: Springer; 2012.
17. Kosuge K, Sato T, Kikukawa N, Takemori M. Morphological Control of rod- and fiberlike SBA-15 type mesoporous silica using water-soluble sodium silicate. Chem Mater. 2004;16:899-905.

18. Spanoudaki A, Albela B, Bonneviot L, Peyrard M. The dynamics of water in nanoporous silica studied by dielectric spectroscopy. Eur Phys J E. 2005;17:21-7.

19. Sliwinska-Bartkowiak M, Dudziak G, Sikorski R, Gras J, Radhakrishnan R, Gubbins KE. Melting/freezing behavior of a fluid confined in porous glasses and MCM-41: dielectric spectroscopy and molecular simulation. J Chem Phys. 2001;114:950-62.

20. Coasne B, Czwartos J, Sliwinska-Bartkowiak M, Gubbins KE Freezing of mixtures confined in silica nanopores: experiment and molecular simulation. J Chem Phys. 2010;133:084701-9.

21. Fedichev PO, Menshikov LI, Bordonskiy GS, Orlov AO. Experimental evidence of the ferroelectric nature of the $\lambda$-point transition in liquid water. JETP Lett. 2011;94:401-5.

22. Chełkowski A. Dielectric physics. North-Holland: Elsevier; 1990.

23. Hoffman JD. Thermal and dielectric study of octamethylcyclotetrasiloxane. J Am Chem Soc. 1953;75(24):6313-4.

24. Sliwinska-Bartkowiak M, Sterczynska A, Long Y, Gubbins KE. Influence of microroughness on the wetting properties of nanoporous silica matrices. Mol Phys. 2014 [Epub ahead of print].

25. Hung FR, Coasne B, Santiso EE, Gubbins KE, Siperstein FR, Sliwinska-Bartkowiak M. Molecular modeling of freezing of simple fluids confined within carbon nanotubes. J Chem Phys. 2005; 122:144706-14.

26. Hung FR, Gubbins KE, Radhakrishnan R, Szostak K, Beguin F, Dudziak G, Sliwinska-Bartkowiak M. Freezing/melting of Lennard-Jones fluids in Carbon Nanotubes. App Phys Lett. 2005;86:103110-3.

27. Pei HR, Yan X, Liu WB, Lan XZ. Phase behavior of tetradecanehexadecane mixtures confined in SBA-15. J Therm Anal Calorim. 2013;112:961-7.

28. Lan XZ, Pei HR, Yan X, Liu WB. Phase behavior of dodecanetetradecane binary system confined in SBA-15. J Therm Anal Calorim. 2012;110:1437-42.

29. Staveley LAK. Phase transitions in plastic crystals. Phys Chem. 1962;13:351-68.

30. Sliwinska-Bartkowiak M, Jazdzewska M, Gubbins KE, Huang L. Melting behavior of bromobenzene within carbon nanotubes. J Chem Eng Data. 2010;55:4183-9.

31. Sliwinska-Bartkowiak M, Jazdzewska M, Huang LL, Gubbins KE. Melting behavior of water in cylindrical pores: carbon nanotubes and silica glasses. Phys Chem Chem Phys. 2008;10:4909-19.

32. Coasne B, Czwartos J, Sliwinska-Bartkowiak M, Gubbins KE. Effect of pressure on freezing of pure fluids and mixtures confined in nanopores. J Phys Chem B. 2009;113:13874-81.

33. Wang LP, Wang TB, Gao CF, Lan X, Lan XZ. Phase behavior of dodecane-hexadecane mixtures in bulk and confined in SBA- 15 . J Therm Anal Calorim. 2014;116:469-76. 